\title{
Differential expression of seven mitochondrion enzymes in osteoarthritis cartilages and normal cartilages
}

\author{
Bipin kumarRai, ${ }^{1}$ Mohd mohasin khan, ${ }^{1}$ Wen-yong Fei, ${ }^{1}$ JI-shan Yuan, ${ }^{1}$ Wei Qian ${ }^{2}$ \\ ${ }^{I}$ Department of Orthopedics, Zhenjiang People's No. 1 Hospital, Zhenjiang, Jiangsu Pr., China, 212000 \\ ${ }^{2}$ Department of E.N.T, Zhenjiang People's No. 1 Hospital, Zhenjiang, Jiangsu Pr., China, 212000 \\ Corresponding to: Wei Qian, dwqian@yahoo.com
}

\begin{abstract}
Osteoarthritis (OA) is an aging-associated rheumatic disease characterized by reduced number of chondrocytes, loss of existing cartilage extracellular matrix, the production of matrix with abnormal composition and pathologic matrix calcification. Recent studies have reported mitochondrial dysfunction played an important role in human $O A$ chondrocytes. Although several lines of evidence support the role for declines in enzyme activity in the etiology of OA, it remains unclear if the expressions of enzymes alter with OA. Here, we determined to quantify the expression of several enzymes in ETC and cytoplasm of mitochondrial in healthy and $O A$ cartilage. These findings suggest that the decreased expression of enzymes in mitochondrial may contribute to $O A$.
\end{abstract}

Keywords: osteoarthritis, cartilage, mitochondrion, enzymes

\section{Introduction}

Osteoarthritis $(\mathrm{OA})$ constitutes a significant public health problem. The prevalence of $\mathrm{OA}$ is greater in women than men, and risk increases with age. it affects all racial and ethnic groups. OA is strongly age-related, it is uncommon before the age of 40 , but the prevalence rises rapidly with age thereafter $(1,2)$. Clinically, OA is characterized by joint pain, credits, swelling, and stiffness, restricted range of motion, fatigue, and functional limitations. Pathological changes can be seen with degradation of the matrix and cell death resulting in the gradual loss of articular cartilage integrity. The chondrocyte is the only cell type that is present in mature cartilage, and it is responsible for repairing the damaged tissue $(3,4)$.

Mitochondria are cellular organelles present in all higher eukaryotes, play a central role in energy metabolism of cells. The dominant role for the mitochondria is the production of ATP by oxidative phosphorylation, as reflected by multienzyme complexes located both in the inner mitochondrial membrane and in the mitochondrial matrix for this task. Electrons that are donated from the Krebs cycle are passed through the electron transport chain (ETC) eventually reducing oxygen and producing water. This potential created by the flux of electrons between the intermembrane space and the matrix is utilized by the ATP synthase to phosphorylate ADP producing ATP(5).

Mitochondrial dysfunctions have been associated with apoptosis, aging, and a number of pathological conditions, including Parkinson disease, diabetes mellitus, Alzheimer disease, and OA(6-8). It was reported decreased mitochondrial bioenergy reserve as a pathogenic factor in degenerative cartilage disease(9). Other research demonstrated that articular chondrocytes from osteoarthritis (OA) showed a significant reduction in complex II and III activities compared to normal chondrocytes, as well as decreased mitochondrial membrane potential $(\triangle \psi \mathrm{m})(10,11)$. However, most studies focused on the activity alteration of mitochondrial respiratory chain complexes in chondrocytes with OA, the expression level alteration of enzymes in ETC are still unclear.

In the present study, we determined to quantify the expression levels of some enzymes in MRC and cytoplasm of mitochondrial in human cartilage from donors with normal and OA cartilage.

\subsection{Material}

\section{Materials and methods}

Antibodies HADHSC :( catalog no.SC-100472), MDHC (H-6) :( catalog no. SC-166879),V-ATPase H :( catalog no. SC-20950), cytochrome b :( catalog no. SC-11436) were all the obtained from Santa cruz company. COX IV antibody (catalog no.4844) was purchased from cell signaling technology. NDUFA1 (catalog no.BA3676), SDH (catalog no. BA3269) were purchased from Boster company. TRIzol reagent was purchased from Invitrogen Life Technologies.

\subsection{Methods}

\subsubsection{Cartilage sample}

Specimens of human articular cartilage were collected from a total of $16 \mathrm{OA}$ patients (three males and six females, 55-74 yr of age) and 12 normal controls (four males and eight females, 42-82 yr of age). OA 
samples were obtained from patients who were under going joint replacement surgery. Normal human cartilage was collected from patients who had amputation after car accident or acute joint trauma. This investigation was approved by the First Peoples' Hospital of Zhenjiang. All patients and relatives provided an informed consent.

\subsubsection{Tissue preparation and staining}

Cartilage were fixed in $4 \%$ paraformaldehyde and embedded in paraffin. The blocks were cut into $4 \mu \mathrm{m}$ slices, heated for $3 \mathrm{~h}$ at $37^{\circ} \mathrm{C}$ in an incubator and then dewaxed and stained with hematoxylin and eosin. One slice from each patient was analyzed under a microscope.

\subsubsection{Western blot analysis}

Cartilage was storage immediately in $-80^{\circ} \mathrm{C}$ when removed until use. Cartilages were cut into small pieces and grinded with liquid nitrogen, then lysed with RIPA. Cartilage lysates were electrophoresed on SDS polyacrylamide gels and transferred onto polyvinylidene difluoride membranes (Perkin Elmer, USA). Membranes were blocked with $5 \%(\mathrm{w} / \mathrm{v})$ BSA in TBST for $1 \mathrm{~h}$ at room temperature and incubated overnight with primary antibodies at $4^{\circ} \mathrm{C}$, followed by HRP-conjugated second antibodies. The immunoreactive bands were detected by chemiluminescence (ECL Plus, Millipore) and relevant blots were quantified by densitometry using the accompanying computerized image analysis program.

\subsubsection{Preparation of RNA and cDNA synthesis}

Cartilages were cut into small pieces and grinded with liquid nitrogen, then lysed with Trizol according to the manufacturer's instructions. Two micrograms of total RNA was reverse transcribed to cDNA in a total reaction volume of $40 \mu \mathrm{l}$ containing $5 \times$ buffer, dNTPs $10 \mathrm{mM}$ each, random hexamers $10 \mu \mathrm{M}$, RNAsin 80 units and 200 units of MMLV reverse transcriptase. Samples were incubated for $10 \mathrm{~min}$ at $25^{\circ} \mathrm{C}, 60 \mathrm{~min}$ at $42^{\circ} \mathrm{C}$, and then stored at $-20^{\circ} \mathrm{C}$.

\subsubsection{RQ-PCR}

Real-time quantitative PCR was performed using EvaGreen dye (BIOTIUM, Hayward, CA, USA) on a 7300 Thermo cycler (Applied Biosystems, Foster City, CA, USA). Real-time fluorescent data were collected and analyzed with SDS 1.3 software (Applied Biosystems, Foster City, CA, USA). PCR was performed in a final volume of $25 \mu \mathrm{l}$, containing $100 \mathrm{ng}$ of cDNA, $0.2 \mathrm{mM}$ of dNTP, $4 \mathrm{mM}$ of $\mathrm{MgCl} 2,0.4 \mu \mathrm{M}$ of primers, 1.2 $\mu \mathrm{l}$ of EvaGreen, 1.0 U of Taq DNA Polymerase. Relative expression levels of the genes were calculated using the $2-\Delta \Delta \mathrm{CT}$ method.

The primers sequences were used as follows:

\begin{tabular}{|l|l|l|}
\hline Primer name & Forward sequence & Reverse sequence \\
\hline NDUFA1 & TTCAAGGACCCAGAAGTAG & GACTCCAGTGATACCCAAA \\
\hline SDHB & ACCTAAAGCACCTGAAGACG & GACTCCAGTGATACCCAAA \\
\hline Cytb & TGGGATTCACGGGACCTT & GCCTTCCTGCTTCATTCG \\
\hline COX & AAAGTGTTGTGAAGAGCGAAGA & TATTCTCAGATTGACGAAG \\
\hline V-ATPase H & GCATAATGGGAGTGTTGA & TATTCTTGGCGAGTTTCT \\
\hline MDH & AGCCGCCTGACCCTCTAT & TCGTGGCATTGGTGTTGA \\
\hline HADHSC & CAGGCAGTTCATGCGTTCC & CGCTATGGTGCTCAGGGT \\
\hline
\end{tabular}

\subsubsection{Statistical analysis}

Statistical analysis was performed using Prism5 (GraphPad Software). All experiments were performed at least in triplicate. Results were expressed as the mean $\pm 95 \%$ confidence interval (CI). Statistical differences between groups were compared By Student's t-test to identify significantly different results. Values of $\mathrm{P}<0.05$ were considered to be statistically significant.

\subsection{Histopathological changes of OA cartilage}

\section{Results}

H\&E staining showed the histological structure of cartilage and excluded the genetic bone and cartilage diseases in normal group. Compared with normal cartilage (fig $2.1 \mathrm{~A}$ and B), synoviocytes proliferation and lymphocytes infiltration could be observed in OA group, even there were patch calcification and fibrosis in some severe patients (Fig 2.1C and D). Two samples with bone and cartilage disease were excluded from normal group. 
A

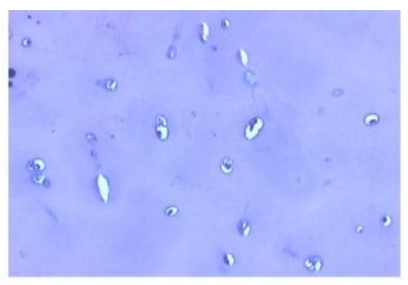

B

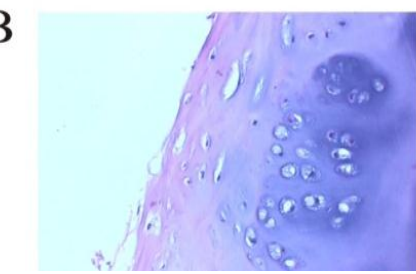

$\mathrm{C}$

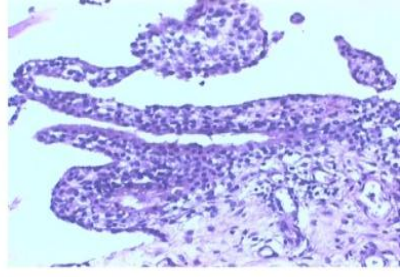

$\mathrm{D}$

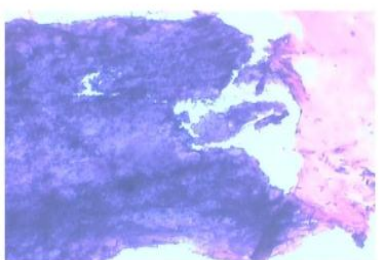

Fig 2.1 HE staining of cartilages in normal and OA groups. A, B: structure of normal cartilage. C: synoviocytes proliferation and lymphocytes infiltration in OA cartilage. D: patch calcification and fibrosis in OA cartilage. $100 \times)$

\subsection{Mitochondrion enzymes changes in OA and normal cartilage using RQ-PCR}

NADH dehydrogenase (ubiquinone) 1 alpha subcomplex 1 (NDUFA1), succinate dehydrogenase complex, subunit B (SDHB), cytochrome b (Cytb), Cytochrome c oxidase ( COX), vacuolar-type H+ATPase (V-ATPase $\mathrm{H})$, malate dehydrogenase $(\mathrm{MDH})$ and hydroxyacyl-Coenzyme A $(\mathrm{CoA})$ dehydrogenase, short chain(HADHSC) were detected by RQ-PCR. The expression levels of NDUFA1 and COX were significantly higher in OA samples compared to normal cartilages, and the difference was significant in COX ( $\mathrm{P}<0.05$ ). Expressions of SDHB, Cytb, V-ATPase and MDH were lower in OA cartilages than in normal cartilages, but the differences were not statistically significant in Cytb, V-ATPase $\mathrm{H}$ and MDH. There was no statistical significance in HADHSC expression level between normal cartilage and OA cartilage (Fig 2.2).
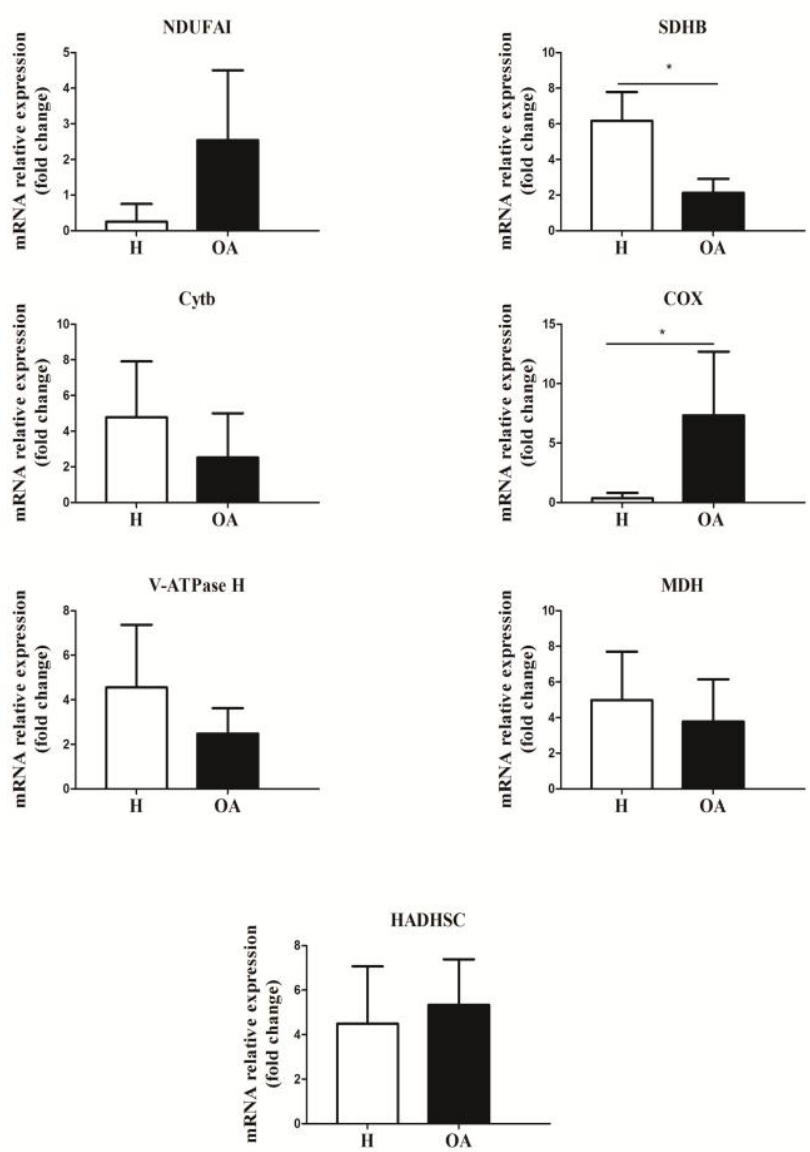
Differential expression of seven mitochondrion enzymes in osteoarthritis cartilages and normal

Fig 2.2 RQ-PCR statistical analysis of the NDUFA1, SDHB, COX, Cytb, V-ATPase H, MDH, and HADHSC enzymes in healthy and $\mathrm{OA}$ cartilages. ABL as a loading control. $\quad(* \mathrm{P}<0.05, * * \mathrm{P}<0.01)$

\subsection{Mitochondrion enzymes changes in $\mathrm{OA}$ and normal cartilage using Western blot assay}

To confirm the RQ-PCR results, we detected the seven enzymes by western blot. The western blot analyses yielded results that were consistent with the RQ-PCR data. The expression levels of NDUFA1 and COX were significantly higher in OA samples compared to normal cartilages, and the difference was significant in COX $(\mathrm{P}<0.05)$. Expressions of SDHB, Cytb, V-ATPase H and MDH were lower in OA cartilages than in normal cartilages, but the differences were not statistically significant in SDHB and MDH. There was no statistical significance in HADHSC expression level between normal cartilage and OA cartilage (Fig 2.3).

A

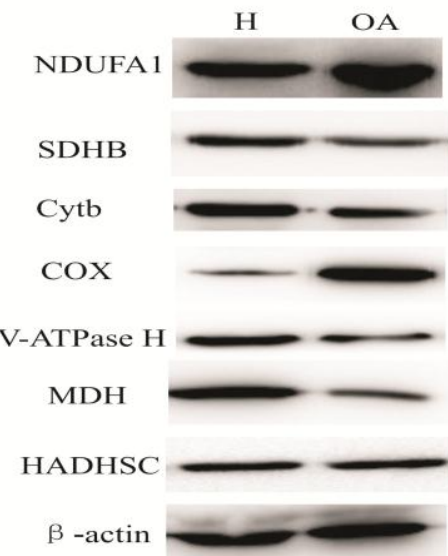

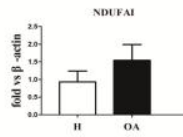
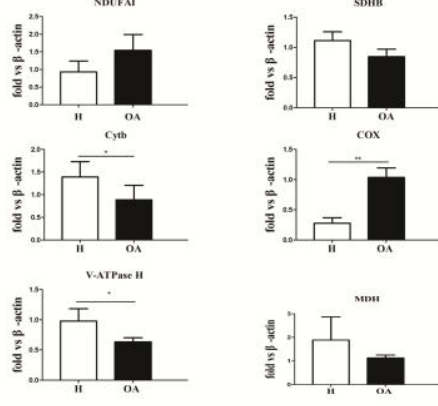

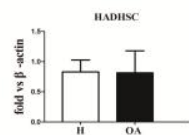

Fig 2.3 Western-blot and statistical analysis of the NDUFA1, SDHB, COX, Cytb, V-ATPase H, MDH, and HADHSC enzymes in healthy and OA cartilages. $\beta$-actin as loading control. A: Western blot results of seven enzymes in normal $(\mathrm{H})$ and $\mathrm{OA}(\mathrm{OA})$ cartilages. B: statistical analysis of seven enzymes in normal $(\mathrm{H})$ and $\mathrm{OA}$

(OA) cartilages. $(* \mathrm{P}<0.05, * * \mathrm{P}<0.01)$

Table 1 Western-blot and RQ-PCR results of NDUFA1, SDHB, COX, Cytb, V-ATPase, MDH and HADHSC in normal and OA cartilages

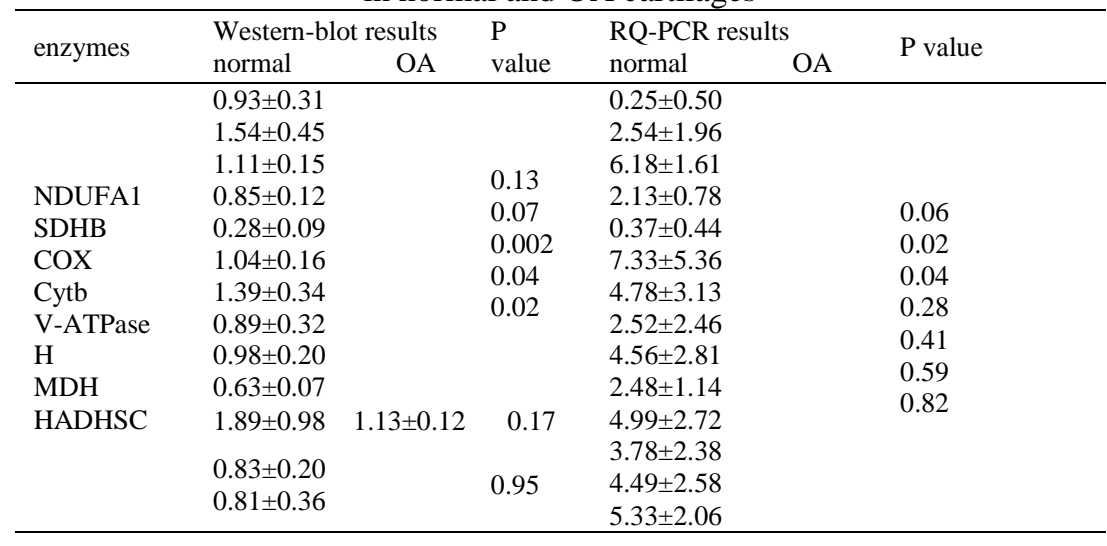

\section{Discussion}

OA is a time or age dependent process leads to aberrant cartilage structure which is characterized by reduced number of chondrocytes, loss of existing cartilage extracellular matrix, the production of matrix with abnormal composition and pathologic matrix calcification. Some studies suggest that the chondrocyte mitochondria are specialized for calcium transport and are important in the calcification of the extracellular matrix. Mineral formation has been demonstrated in matrix vesicles (MV) and within mitochondria. Direct suppression of mitochondrial respiration promoted MV-mediated mineralization in chondrocytes(12-14). In this study, H\&E staining showed matrix calcification exist in most of patients with OA cartilage, and we also found some key enzymes of mitochondria changed in these cartilages in subsequent studies, suggesting that these alteration in the mitochondria enzymes may also be associated with matrix calcification. While, this assumption requires further studies.

The mitochondrial ATP production relies on the electron transport chain (ETC), composed of respiratory chain complexes NADH-coenzyme Q1 reductase (complex I), SDH (complex II), ubiquinone cytochrome c reductase ( complex III), Cytochrome c oxidase ( complex IV) and ATPase ( complex V). Most previous studies 
focused on electron transport complex activity changes in articular chondrocytes from osteoarthritis (OA) compared to normal chondrocytes, but the expression of ETC and other important enzymes in cytoplasm of mitochondrial in OA chondrocytes are still unclear until now. It was reported that complex II and III activities were significantly lower in OA than in normal chondrocytes. Complex I mean values in OA chondrocytes were lower than in normal cells, but the difference was not statistically significant. The activity of complex IV was similar in the two groups. However, the mitochondrial mass was increased in OA(7). In this study, we compared expression levels of some enzymes in ETC and found that NDUFA1 and COX increased in OA cartilages, while SDHB, Cytb and V-ATPase H decreased in OA cartilages, these changes were similar as the changes of ETC activity. COX is an important structural protein in mitochondrial, it can be used effectively as a mitochondrial loading control. The COX expression increased in OA cartilages, it maybe suggested that the mitochondrial mass was increased in OA. This might be a compensatory mechanism to offset the deficiency in electron transport chain. Expression levels of SDHB, Cytb and V-ATPase H decreased in OA cartilages, we hypothesized that ETC protein decline contribute to its dysfunction, lead to low ATP production and high production of oxygen free radicals, thereby accelerating OA process. It was reported that a dysfunction in complexes II, III and IV could be solved by over-loading the electron transport via complex I, a pathway with little oxygen consumption(15), this may be one reason for increased expression of NDUFA1.

Cytosolic malate dehydrogenase (MDHC) is an important NAD-dependent enzyme involved in glycometabolism that catalyzes the formation of oxaloacetate and NADH from L-malate and NAD. It's located in matrix of mitochondria, is a symbol enzyme of matrix. We found MDH was lower in OA cartilage, we hypothesized that MDH decline might also contribute to mitochondrial dysfunction. HADHSC (hydroxyacylCoenzyme $\mathrm{A}(\mathrm{CoA})$ dehydrogenase, short chain) is also a mitochondrial matrix protein which exists as a homodimer that participates in lipid metabolism and is essential for the $\int$-oxidation of medium and short chain fatty acids. More specifically, HADHSC catalyzes the dehydrogenation of 3-hydroxyacyl-CoAs to their corresponding 3-ketoacyl-CoAs while NAD+ is simultaneously reduced to NADH. Very few studies have been performed to determine the value of fatty acids for energy generation by cartilage slices or cultured cells. It is likely that chondrocytes can metabolize fatty acids as an energy source; however the importance of these compounds in the overall energy economy of the tissue has not been determined. While, in this study we found no statistically significance of HADHSC between cartilages and normal cartilages, this may indicate HADHSC not play an important role in pathopoiesia of OA.

\subsection{Conclusions}

\section{Chapter 5 Conclusion}

In conclusion, our present study suggests that expression levels of some mitochondrion key enzymes of MRC and matrix changed in OA cartilages compare with normal cartilages. These changes may contribute to mitochondrion dysfunction and play an important role in pathopoiesis of OA.

\section{Reference}

[1]. Lotz M: Osteoarthritis year 2011 in review: biology. Osteoarthritis and cartilage / OARS, Osteoarthritis Research Society 20: 192196, 2012.

[2]. Loeser RF: Aging and osteoarthritis. Current opinion in rheumatology 23: 492-496, 2011.

[3]. Vaamonde-Garcia C, Riveiro-Naveira RR, Valcarcel-Ares MN, Hermida-Carballo L, Blanco FJ and Lopez-Armada MJ: Mitochondrial dysfunction increases inflammatory responsiveness to cytokines in normal human chondrocytes. Arthritis and rheumatism 64: 2927-2936, 2012.

[4]. Loeser RF: Aging and osteoarthritis: the role of chondrocyte senescence and aging changes in the cartilage matrix. Osteoarthritis and cartilage / OARS, Osteoarthritis Research Society 17: 971-979, 2009.

[5]. Desler C, Hansen TL, Frederiksen JB, Marcker ML, Singh KK and Juel Rasmussen L: Is There a Link between Mitochondrial Reserve Respiratory Capacity and Aging? Journal of aging research 2012: 192503, 2012.

[6]. Schapira AH: Mitochondrial disease. Lancet 368: 70-82, 2006

[7]. Maneiro E, Martin MA, de Andres MC, et al:: Mitochondrial respiratory activity is altered in osteoarthritic human articular chondrocytes. Arthritis and rheumatism 48: 700-708, 2003.

[8]. Harrison C: Obesity and diabetes: Mitochondrial uncoupler blocks metabolic disease. Nature reviews 13: 20, 2013

[9]. Johnson K, Jung A, Murphy A, Andreyev A, Dykens J and Terkeltaub R: Mitochondrial oxidative phosphorylation is a downstream regulator of nitric oxide effects on chondrocyte matrix synthesis and mineralization. Arthritis and rheumatism 43: 1560-1570, 2000.

[10]. Terkeltaub R, Johnson K, Murphy A and Ghosh S: Invited review: the mitochondrion in osteoarthritis. Mitochondrion 1: 301-319, 2002.

[11]. Johnson K, Svensson CI, Etten DV, et al.: Mediation of spontaneous knee osteoarthritis by progressive chondrocyte ATP depletion in Hartley guinea pigs. Arthritis and rheumatism 50: 1216-1225, 2004.

[12]. Blanco FJ, Lopez-Armada MJ and Maneiro E: Mitochondrial dysfunction in osteoarthritis. Mitochondrion 4: 715-728, 2004.

[13]. Blanco FJ, Rego I and Ruiz-Romero C: The role of mitochondria in osteoarthritis. Nature reviews 7: 161-169.

[14]. Cillero-Pastor B, Rego-Perez I, Oreiro N, Fernandez-Lopez C and Blanco FJ: Mitochondrial respiratory chain dysfunction modulates metalloproteases $-1,-3$ and -13 in human normal chondrocytes in culture. BMC musculoskeletal disorders 14: 235

[15]. Liu JT, Guo X, Ma WJ, et al.: Mitochondrial function is altered in articular chondrocytes of an endemic osteoarthritis, Kashin-Beck disease. Osteoarthritis and cartilage / OARS, Osteoarthritis Research Society 18: 1218-1226. 